Jorgete Maria Buso Bazzo

Elizabeth Milla Tambara ${ }^{1}$

Antonio CARlos Ligocki CAMPOS ${ }^{1}$

Rodrigo de Paula Feljó ${ }^{2}$

Artigo Original

Palavras-chave

Cesárea

Cicatriz/ultrassonografia Metrorragia/ultrassonografia Útero/anatomia e histologia

Útero/ultrassonografia

Keywords

Cesarean section Cicatrix/ultrasonography Metrorrhagia/ultrasonography

Uterus/anatomy e histology

Uterus/ultrasonography

\section{Avaliação ultrassonográfica de cicatriz uterina pós-cesariana segmentar transversa}

\section{Ultrasound evaluation of uterine scar after segmental transverse}

\author{
cesarean surgery
}

\section{Resumo}

OBJETIVO: Avaliar a medida do segmento uterino inferior pela ultrassonografia transvaginal em um grupo de mulheres não grávidas e descrever os achados morfológicos na cicatriz daquelas submetidas à cesárea. MÉTODOS: Estudo retrospectivo para o qual foram avaliadas 155 imagens de ultrassonografias transvaginais obtidas de mulheres no menacme, não grávidas. Os exames foram realizados entre janeiro de 2008 e novembro de 2011 . Foram selecionados três grupos: mulheres que nunca ficaram grávidas (Grupo Controle I), mulheres com partos vaginais anteriores (Grupo Controle II) e mulheres com cesárea prévia (Grupo de Estudo). Foram excluídas as mulheres com útero em retroflexão, usuárias de dispositivo intrauterino, gestantes e mulheres com menos de um ano do último evento obstétrico. Os dados obtidos foram submetidos à análise estatística com o programa Statistica ${ }^{\circledR}$, versão 8.0. Para a comparação dos grupos em relação às variáveis quantitativas foram utilizados os testes ANOVA e LSD. Para a comparação entre a espessura do istmo anterior e posterior utilizou-se o teste $t$ de Student. Para a associação entre variáveis quantitativas estimou-se o coeficiente de correlação de Spearman. Valores p<0,05 indicaram significância estatística. RESULTADOS: Houve diferença significativa entre as espessuras do istmo anterior e posterior do útero apenas no grupo de mulheres com cesárea anterior. Na comparação dos grupos dois a dois, não houve diferença significativa entre a espessura do istmo posterior e anterior nos Grupos Controle, mas essa diferença foi significativa quando comparado o Grupo de Estudo com cada Grupo Controle. No Grupo de Estudo, não foi encontrada correlação entre a espessura do istmo anterior e o número de cesáreas ou ao tempo decorrido desde o último parto. A presença de lesão em cunha foi observada na cicatriz de cesárea em 30,6\% das mulheres do Grupo de Estudo, das quais 93\% apresentavam queixa de sangramento pós-menstrual. CONCLUSÃO: A avaliação da relação entre a espessura da parede anterior e parede posterior do segmento inferior uterino pela ultrassonografia transvaginal é um método adequado para a avaliação em mulheres com cesáreas prévias.

\section{Abstract}

PURPOSE: To evaluate the thickness of the lower uterine segment by transvaginal ultrasound in a group of non-pregnant women and to describe the morphologic findings in the scar of those submitted to cesarean section. METHODS: A retrospective study of 155 transvaginal ultrasound images obtained from premenopausal and non-pregnant women, conducted between January 2008 and November 2011 . the subjects were divided into three groups: women who were never pregnant (Control Group I), women with previous vaginal deliveries (Control Group II) and women with previous cesarean section (Observation Group). We excluded women with a retroverted uterus, intrauterine device users, pregnant women and those with less than one year of tsince the last obstetrical event. The data were analyzed statistically with Statistica ${ }^{\circledR}$, version 8.0 software. ANOVA and LSD were used to compare the groups regarding quantitative variables and the Student's ttest was used to compare the thickness of the anterior and posterior isthmus. The Spearman correlation coefficient was calculated to estimate the association between quantitative variables. P values $<0.05$ were considered statistically significant. RESULTS: There was significant difference between the thickness of the anterior and posterior isthmus only in the group of women with previous cesarean section. Comparing the groups two by two, no
Correspondêncio

Jorgete Maria Buso Bazzo Departamento de Tocoginecologia do Hospital de Clínicas do Universidade Federal do Paran Rua General Carneiro 181 - Centro CEP: $80060-900$ Curitiba (PR), Brasi

Recebido 05/03/2012

Aceito com modificacōes 02/04/2012
1 Programa de Pós-graduação em Clínica Cirúrgica da Universidade Federal do Paraná - UFPR - Curitiba (PR), Brasil. 2 Departamento de Tocoginecologia do Hospital de Clínicas da Universidade Federal do Paraná - UFPR - Curitiba (PR), Brasil. Conflito de interesses: não há 
significant differences between the thickness of the anterior and posterior isthmus were observed in the Control Groups, but this difference was significant when we compared the Observation Group with each Control Group. In the Observation Group, no correlation was found between the thickness of the isthmus and the number of previous cesarean deliveries or the time elapsed since the last birth. A niche was found in the cesarean scar in $30.6 \%$ of the women in the Observation Group, 93\% of whom complained of post-menstrual bleeding. CONCLUSION: The relationship between the thickness of the anterior and posterior wall of the lower uterine segment by transvaginal ultrasound is a suitable method for the evaluation of the uterine lower segment in women with previous cesarean sections.

\section{Introdução}

Após a introdução da técnica da cesariana segmentar transversa baixa na rotina obstétrica, a frequência desse procedimento em relação ao parto vaginal começou a se elevar, como em todo o mundo, atingindo 30,2\% do total de partos em $2005^{1}$, embora a Organização Mundial de Saúde recomende uma taxa de cesáreas entre 10 e $15 \%{ }^{2}$. No Brasil, em 2010 , pela primeira vez, o percentual de cesarianas superou o de partos normais, chegando a $52 \%$ do total ${ }^{3}$.

Embora seja um procedimento relativamente seguro pelo aperfeiçoamento das técnicas cirúrgicas e anestésicas, a cesárea está associada a riscos de longo prazo, tais como aderências pélvicas pós-operatórias, rotura uterina, placenta prévia e acretismo placentário, e esses riscos se elevam significativamente no caso de cesáreas múltiplas ${ }^{4}$.

O fato de os primeiros estudos detalhados sobre placenta prévia e acretismo placentário terem surgido duas décadas após a introdução da cesárea na rotina obstétrica, é altamente sugestivo de uma relação direta entre cesárea anterior e inserção placentária anormal. Estudos epidemiológicos recentes têm demonstrado que o fator de risco mais importante para placenta prévia e acreta é a cesárea prévia, sugerindo que uma falha na decidualização na área da cicatriz pode ter impacto significativo sobre a implantação e o desenvolvimento placentário 5 .

Embora esses riscos sejam bem conhecidos, os efeitos a longo prazo decorrentes da cesárea têm sido pouco estudados e só recentemente surgiram alguns estudos com a finalidade de avaliar a integridade da região uterina com cicatrizes de cesáreas prévias ${ }^{6,7}$. Vários métodos de imagem têm sido utilizados nessa investigação, como a histerografia, histerossonografia, ressonância magnética e a ultrassonografia ${ }^{7}$.

Por tratar-se de método seguro e não invasivo, a ultrassonografia tem sido o método de eleição utilizado nessa investigação. Foi com a ultrassonografia que alguns estudos identificaram uma área anecoica triangular na região da incisão cirúrgica, denominada "nicho", "divertículo" ou "cunha", que pode conter endométrio em seu interior e ser responsável por sangramento uterino anormal ${ }^{6-9}$, gestação ${ }^{10}$ ou endometriose ${ }^{11}$ em cicatriz de cesárea.

Além desses achados, alguns autores observaram uma redução significativa da espessura miometrial no local da cicatriz que, em alguns casos, pode representar mais que
$50 \%$ de perda da camada do miométrio e elevar o risco de rotura ou deiscências uterinas, acretismo placentário ou placenta prévia em gestações futuras ${ }^{4,12,13}$.

Diversos métodos têm sido propostos para avaliação ultrassonográfica da espessura miometrial em mulheres submetidas à cesárea, mas ainda não se obteve um consenso na literatura. A maioria dos estudos tem utilizado a comparação entre a espessura do miométrio na região da cicatriz com o miométrio normal adjacente cranial $^{14,15}$ ou caudal ${ }^{4,9}$, medido no plano longitudinal, considerando que valores inferiores a $3,5 \mathrm{~mm}$ indicam uma cicatriz gravemente defeituosa ${ }^{12,13,16}$. No entanto, a reprodutibilidade da maioria desses estudos é baixa, e várias alternativas, como avaliar a espessura total do segmento inferior uterino ${ }^{17}$ ou utilizar apenas a mensuração de sua camada muscular ${ }^{18}$, têm sido propostas para melhorar a acurácia do método.

Embora a espessura miometrial seja inversamente proporcional à idade, não há diferenças significativas entre a parede anterior e a parede posterior, que possuem espessuras semelhantes ${ }^{19}$. Com base nesse parâmetro, este estudo propõe comparar a espessura das paredes anterior e posterior uterina em um grupo de mulheres não grávidas pela ultrassonografia transvaginal e descrever os achados morfológicos resultantes de cicatriz no segmento inferior uterino daquelas submetidas à cesárea. Os objetivos do estudo são avaliar a existência de diferença entre a espessura da região ístmica uterina anterior e a posterior nas pacientes submetidas a cesariana, se há correlação entre a diferença de espessura e o número de cesáreas, se há correlação entre a diferença de espessura e o tempo de evolução após a última cirurgia e se o método proposto constitui um critério adequado para a avaliação do segmento inferior uterino nas mulheres com história de cesáreas prévias.

\section{Métodos}

Foi desenvolvido um estudo retrospectivo de imagens de ultrassonog rafias transvaginais obtidas de mulheres no menacme, não grávidas, encaminhadas para realização de ultrassonografia por qualquer indicação ginecológica. Foram incluídas no estudo as imagens de mulheres no menacme, não gestantes, com útero em anteversoflexão e 
período superior a um ano do último evento obstétrico. Foram excluídas imagens de mulheres com lesões uterinas, útero em retroflexão, usuárias de dispositivo intrauterino e com cirurgias uterinas anteriores. Após a aplicação dos critérios de inclusão e exclusão, foram selecionadas as imagens de 155 mulheres atendidas no período de janeiro de 2008 e novembro de 2011, numa clínica de ecografia de Curitiba, Paraná.

As imagens foram subdivididas em três grupos: Grupo Controle I - imagens ultrassonográficas de mulheres sem história de gestação, sem patologias uterinas e livres de quaisquer operações sobre o útero; Grupo Controle II imagens ultrassonográficas de mulheres com história de parto vaginal sem intercorrências, na ausência de quaisquer intervenções sobre o útero. Esse grupo destinava-se ao estudo do efeito da gestação sobre as dimensões das paredes anterior e posterior uterina na junção corpo/istmo; e Grupo de Estudo - imagens ultrassonográficas de mulheres com história de uma ou mais cesáreas, tendo decorrido mais de um ano da última intervenção, sem outras cirurgias uterinas.

Todas as mulheres foram submetidas à ultrassonografia transvaginal da cérvix, útero e anexos pelo mesmo ultrassonografista. Foi utilizado equipamento da marca Siemens, modelo Sonoline SI250, com imagem em tempo real, modo $\mathrm{B}$, e transdutor setorial endocavitário de 6,5 $\mathrm{MHz}$. As medidas foram efetuadas com medidores eletrônicos (calipers) na tela do equipamento.

Inicialmente, utilizando-se o plano sagital, foi visualizado o fundo uterino, identificada sua posição e a regularidade de seus contornos, sendo efetuada a seguir a mensuração dos seus três diâmetros. Mediu-se, então, a espessura máxima do endométrio em corte sagital.

O útero foi examinado no plano longitudinal e o orifício interno cervical foi identificado como o ponto de junção entre a cavidade endometrial e o canal cervical. A flexão uterina foi determinada pela angulação entre o eixo longitudinal uterino e o eixo longitudinal da cérvix. A anteversoflexão uterina foi determinada quando o maior eixo do corpo uterino estava desviado anteriormente em relação ao maior eixo da cérvix.

A observação da cicatriz de cesárea pela ultrassonografia transvaginal se iniciou pela visualização de uma linha hiperecoica acima do nível do orifício interno, estendendo-se para a interface vesicouterina no plano sagital. A mensuração das paredes anterior e posterior uterina foi realizada na altura da cicatriz.

As Figuras 1 e 2 mostram como foi efetuada a mensuração dos istmos anterior e posterior. As setas apontam para as medidas efetuadas pelos calipers.

Defeitos na cicatriz foram considerados quando uma imagem hipoecoica foi observada no istmo anterior e foram medidos em duas dimensões no plano sagital (anteroposterior e cefalocaudal) e transversamente no plano coronal. No caso de cesáreas múltiplas, buscou-se identificar todas as cicatrizes, e a espessura do miométrio na cicatriz mais distal em relação ao orifício cervical interno foi considerada como representativa daquela paciente.

Nos casos em que foi visualizada a presença de bolsa em forma de cunha na parede anterior uterina, na topografia da cicatriz de cesárea, a quantificação do espaço hipoecoico foi avaliada pela medida dos três diâmetros para obter o volume da coleção. Todos os exames foram registrados em filme e arquivados.

O pesquisador procedeu a revisão dos prontuários e das imagens ultrassonográficas para a confirmação dos critérios de inclusão e exclusão, coleta da história obstétrica e das mensurações ultrassonográficas efetuadas. Os dados obtidos foram registrados pelo pesquisador de forma padronizada na Ficha de Coleta de Dados. A ficha continha as informações sobre a idade, partos vaginais, cesáreas, tempo decorrido desde o último evento obstétri$\mathrm{co}$, medida do istmo anterior uterino e medida do istmo posterior uterino. A relação entre a espessura das paredes uterinas foi calculada posteriormente pelo pesquisador $\mathrm{e}$ inserida na ficha de coleta de dados.

Os dados foram digitados em planilha eletrônica (Microsoft Excel ${ }^{\circledR}$ ), conferidos e exportados para posterior análise estatística com o programa computacional Statistica ${ }^{\circledR}$, versão 8.0.

Para a comparação dos três grupos em relação às variáveis quantitativas, foi considerado o modelo de análise de variância com um fator (ANOVA) e o teste LSD (least significant difference). Para a comparação entre a espessura do istmo anterior e istmo posterior, dentro de cada grupo, foi considerado o teste $t$ de Student para amostras pareadas. Para a avaliação da associação entre variáveis quantitativas, estimou-se o coeficiente de correlação de Spearman. Valores $\mathrm{p}<0,05$ indicaram significância estatística.

Por tratar-se de estudo retrospectivo foi solicitado e concedida a dispensa do preenchimento do Termo de Consentimento Livre e Esclarecido. Para preservar a confidencialidade das pacientes, foram utilizadas suas iniciais na Ficha de Coleta de Dados.

O protocolo do estudo foi aprovado pelo Comitê de Ética em Pesquisa do Hospital de Clínicas da Universidade Federal do Paraná sob registro CEP no 2646253/2011-11.

\section{Resultados}

A idade das participantes do estudo variou de 15-53 anos, com mediana de 37,1 anos. O Grupo Controle I era constituído por 41 pacientes, o Grupo Controle II por 16 pacientes e o Grupo de Estudo 98 pacientes, sendo que 85 delas haviam tido apenas cesáreas e 13 haviam tido cesáreas e partos normais. Tanto no Grupo Controle II quanto no 
Tabela 1. Medida da espessura e diferença absoluta entre as espessuras do istmo anterior e posterior nos três grupos

\begin{tabular}{|c|c|c|c|c|c|}
\hline Grupo & Variável & n & Média & $\begin{array}{l}\text { Desvio } \\
\text { padrão }\end{array}$ & Valor $p$ \\
\hline \multirow{3}{*}{ Controle I } & Istmo anterior & 41 & 9,6 & 1,6 & \multirow{3}{*}{0,3} \\
\hline & Istmo posterior & 41 & 9,4 & 1,6 & \\
\hline & $\begin{array}{c}\text { Diferença } \\
\text { (posterior - anterior) }\end{array}$ & 41 & $-0,2$ & 1,3 & \\
\hline \multirow{3}{*}{ Controle II } & Istmo anterior & 16 & 11,0 & 2,4 & \multirow{3}{*}{0,1} \\
\hline & Istmo posterior & 16 & 12,0 & 2,3 & \\
\hline & $\begin{array}{c}\text { Diferença } \\
\text { (posterior - anterior) }\end{array}$ & 16 & 0,6 & 1,9 & \\
\hline \multirow{3}{*}{ Grupo de Estudo } & Istmo anterior & 98 & 6,7 & 2,2 & \multirow{3}{*}{$<0,001$} \\
\hline & Istmo posterior & 98 & 13,4 & 2,8 & \\
\hline & $\begin{array}{c}\text { Diferença } \\
\text { (posterior - anterior) }\end{array}$ & 98 & 6,8 & 3,2 & \\
\hline
\end{tabular}

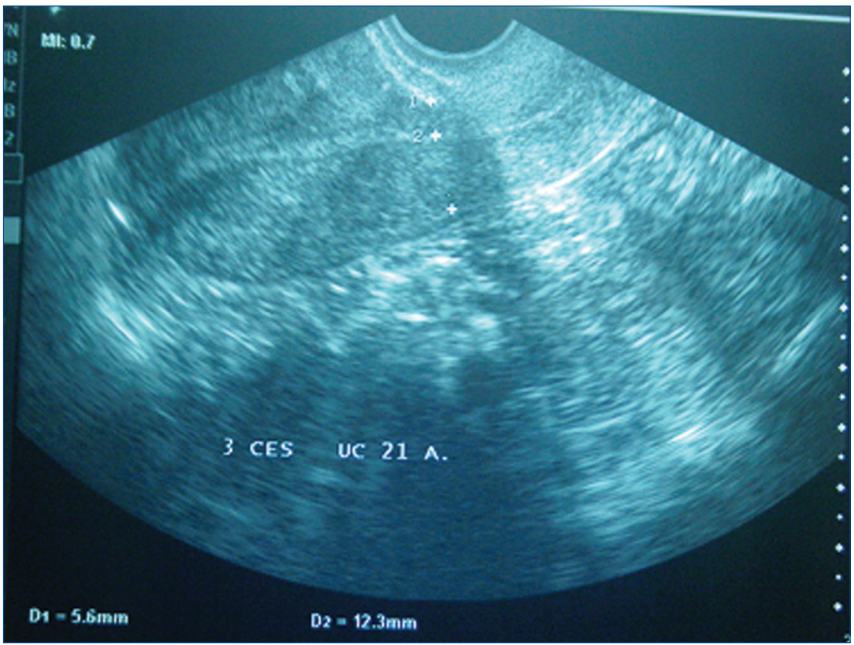

Figura 1. Exemplo de medidas do istmo anterior e posterior semelhantes em paciente que teve apenas partos vaginais (Grupo Controle II)

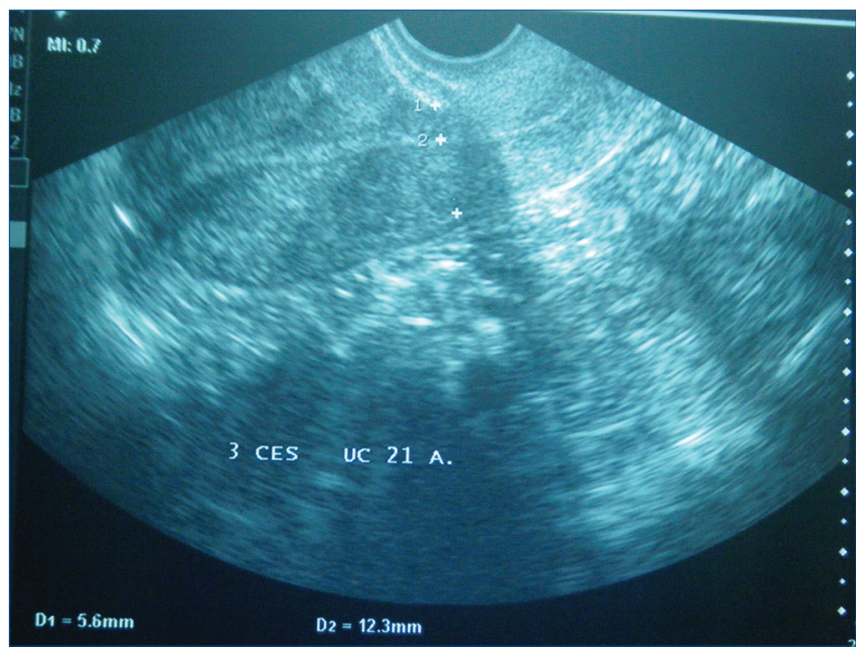

Figura 2. Exemplo de medidas do istmo anterior e posterior em paciente com três cesáreas (Grupo de Estudo)

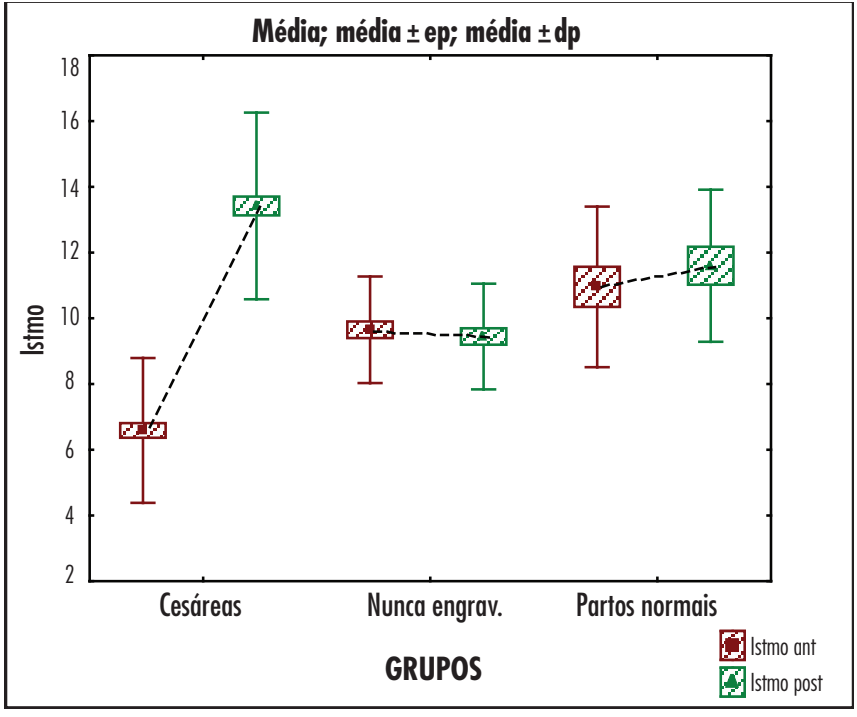

Figura 3. Comparação da espessura do istmo anterior e posterior nos três grupos. A espessura do istmo anterior e posterior é semelhante nas mulheres do Grupo Controle, ao passo que a diferença de espessura entre as paredes anterior e posterior é significativa nas mulheres com história de cesárea

Grupo de Estudo, o número de gestações variou entre 1 e 3. Entre as mulheres do Grupo Controle II, a última gestação havia ocorrido entre 1 e 24 anos. No Grupo de Estudo, a última gestação ocorreu entre 1 e 25 anos. No Grupo de Estudo, 28 mulheres apresentavam queixa de pequena perda de sangue pós-menstrual. Essa queixa não foi relatada nas pacientes dos Grupos Controle I e II.

Houve diferença significativa entre as espessuras do istmo anterior e posterior apenas no grupo de mulheres com cesárea anterior. Nos Grupos Controle I e II as diferenças observadas não foram significativas.

$\mathrm{Na}$ Tabela 1 são apresentadas as estatísticas descritivas das espessuras e da diferença absoluta entre as espessuras do istmo anterior e posterior. Também são apresentados os valores p dos testes estatísticos.

Na comparação dos grupos dois a dois, os resultados mostram que não há diferenças significativas para a média da diferença da espessura do istmo posterior e anterior nos Grupos Controle. No entanto, houve diferença significativa na comparação entre as mulheres que nunca engravidaram e as que tiveram cesáreas (Grupo Controle I x Grupo de Estudo) e entre as mulheres que só tiveram partos normais e as que tiveram cesáreas (Grupo Controle II x Grupo de Estudo). O resultado da comparação do istmo anterior e posterior entre os grupos pode ser visualizado na Figura 3.

Em relação ao número de cesáreas, a espessura média do istmo anterior foi de 7,0 $\mathrm{mm}$ nas mulheres com uma cesárea, de 6,5 mm nas mulheres com 2 cesáreas e de $5,7 \mathrm{~mm}$ nas mulheres com 3 cesáreas, o que indica que não foram encontradas diferenças significativas na espessura média do istmo anterior em relação ao número de cesáreas 

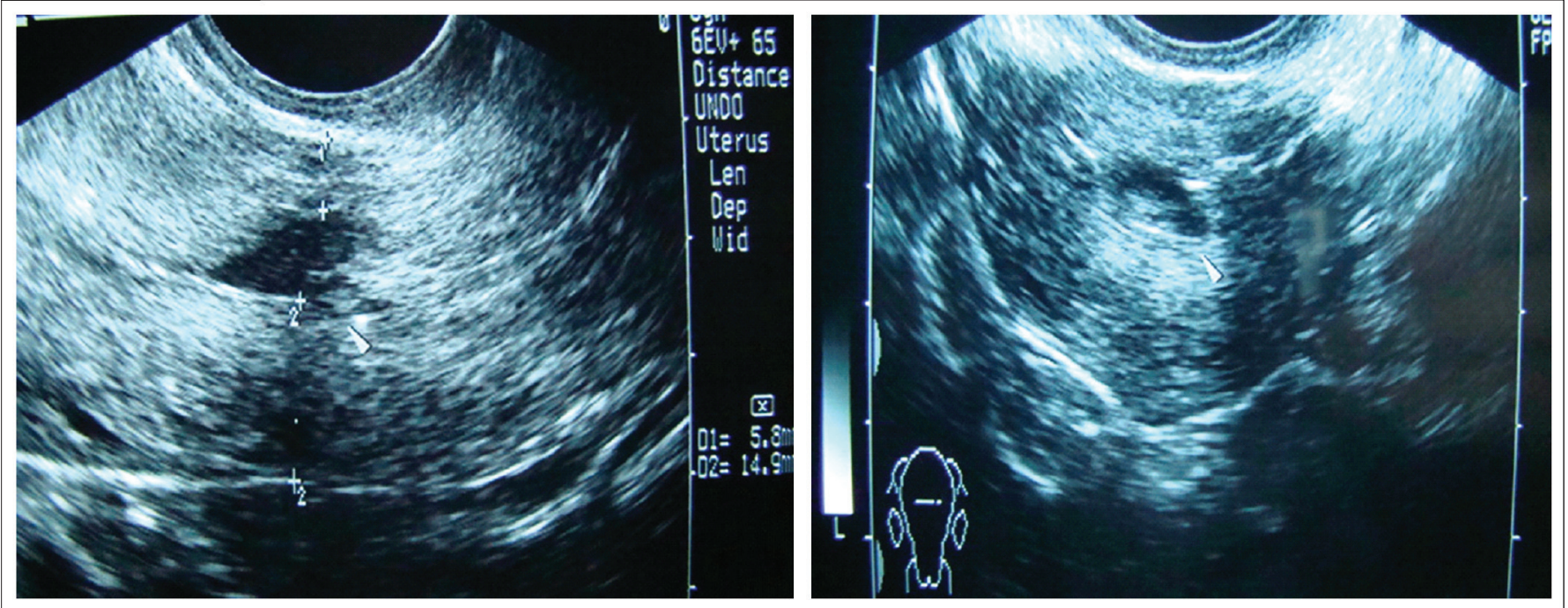

Figura 4. Paciente no sexto dia do ciclo menstrual. Lesão em cunha, com conteúdo espesso na cavidade da cunha em corte longitudinal (a) e corte transversal do útero (b)

$(\mathrm{p}=0,2)$, embora haja uma tendência para a redução da espessura quanto maior for o número de cesáreas.

Também não foi observada associação entre o tempo decorrido desde a última cesárea e a espessura do istmo anterior $(\mathrm{p}=0,683)$.

A presença de lesão em cunha foi observada em 30,6\% das mulheres do Grupo de Estudo. Verificou-se que nas mulheres com lesão de cunha a espessura média do istmo anterior é de $5,1 \mathrm{~mm}$, enquanto a espessura média do istmo posterior é de $13,7 \mathrm{~mm}$, mostrando que existe diferença estatisticamente significativa entre a espessura do istmo anterior e posterior $(\mathrm{p}<0,001)$.

Entre essas pacientes foi observado que, em média, a espessura do istmo posterior é o dobro da espessura do istmo anterior, como pode ser visualizado na Figura 2.

Das 30 pacientes que apresentavam lesão de cunha ao exame ultrassonográfico, 28 tinham queixa de pequena perda de sangue pós-menstrual (93\%), o que corresponde a 28,5\% do total de mulheres com história de cesárea (Figura 4).

\section{Discussão}

Com o crescente número de cesáreas em todo o mundo, a investigação da cicatriz uterina de cesárea adquiriu uma maior importância na obstetrícia e na ginecologia. Isso ocorre pelo fato de a cicatriz poder alterar a anatomia local e comprometer o futuro, predispondo as pacientes a condições como placenta prévia, acretismo placentário, rotura uterina, gestação ectópica no nicho da cicatriz ou sangramento uterino pós-menstrual anormal ${ }^{4,5,7}$.

A ultrassonografia, tanto por via transvaginal quanto por via transabdominal, é o método utilizado para avaliar a cicatriz de cesárea e a medida da espessura do segmento uterino inferior na maioria dos estudos publicados. Embora exista uma forte correlação entre os dois métodos, estudos recentes sugerem que a via transvaginal apresenta melhor reprodutibilidade entre os observadores ${ }^{13,16,17}$, razão pela qual essa foi a via escolhida para este estudo.

$\mathrm{Na}$ literatura, duas variáveis aparecem associadas a deficiências na cicatriz: a história de cesáreas múltiplas e o útero em retroflexão. A chance de uma mulher com útero em retroflexão apresentar uma cicatriz deficiente é cerca de duas vezes maior do que nas mulheres com útero em anteroflexão, e a razão para que isso ocorra é que, na retroflexão, o útero está sob um grau de tensão que pode comprometer a cicatrização cirúrgica ${ }^{4}$. Por esse motivo, o útero retrofletido foi utilizado como critério de exclusão neste estudo.

A literatura propõe a medida ultrassonográfica do segmento uterino inferior na gestação de mulheres com cesárea prévia por considerar a existência de uma correlação inversa entre a espessura desse segmento e o risco de deficiência na cicatriz ${ }^{1,12,20}$. Neste estudo, em concordância com outros autores ${ }^{4,14}$, foi avaliada uma amostra de mulheres não grávidas por entender que a detecção ultrassonográfica de defeitos na cicatriz uterina é mais fácil de ser visualizada em mulheres não gestantes.

Neste estudo, verificou-se a redução da espessura do miométrio em 30,6\% das mulheres com história de cesárea, e, em 5,1\% delas, essa redução na altura da cicatriz de cesárea era superior a $50 \%$. Ainda, a espessura do istmo anterior em $5,1 \%$ das mulheres desse grupo era inferior a $3,5 \mathrm{~mm}$. Esses achados são concordantes com a literatura e indicativos de cicatrizes com deficiências graves ${ }^{4,12,13,16}$, elevando o risco para rotura, deiscência uterina, acretismo ou gravidez na cicatriz cirúrgica ${ }^{4,5,10,12}$.

A presença de defeito triangular anecoico na parede anterior uterina, entre o corpo e a cérvix na cicatriz de cesárea e 
sob a forma de "cunha" é relatada por vários autores ${ }^{4,6,9,21}$ e sua incidência varia entre 19,4 e $56 \%$ nos diversos estudos ${ }^{4,6}$. Em cerca de 30-40\% dessas mulheres há queixa de sangramento ou spotting pós-menstrual, que é diretamente proporcional à gravidade do defeito ${ }^{6,21}$. Neste estudo, encontrou-se que 30,6\% das mulheres com cesárea prévia apresentavam lesão em cunha e destas $93 \%$ referiam sangramento pós-menstrual de pouca monta, normalmente de tonalidade marrom, achados concordantes com a literatura.

Além do sangramento pós-menstrual, outros sintomas clínicos também foram associados aos defeitos na cicatriz de cesárea, como a dismenorreia, a dor pélvica crônica e a dispareunia ${ }^{15}$. Entretanto, as pacientes deste estudo não foram arguidas em relação a essa sintomatologia.

A maior parte dos estudos nos quais se avaliou a redução da espessura do miométrio em pacientes submetidas à cesárea, comparou-se o miométrio na região da cicatriz do istmo anterior com o miométrio normal adjacente, superior ou inferior ${ }^{4,9,14,15,17,18}$. Há apenas um estudo que investigou a relação existente entre a espessura das paredes anterior e posterior uterina, e nele, os autores concluíram que a espessura miometrial sofre uma redução global com o avanço da idade, mas a relação entre as espessuras das paredes anterior e posterior é semelhante em qualquer faixa etária ${ }^{19}$. Por essa razão utilizou-se neste estudo a comparação da espessura do istmo anterior e posterior, o que, em tese, permitiria uma melhor quantificação da redução miometrial na altura da cicatriz uterina.

Neste estudo, observou-se que a espessura do istmo posterior e anterior nas mulheres que tiveram apenas partos normais e naquelas que nunca engravidaram era semelhante, sendo que, na metade das mulheres de ambos os grupos, o istmo anterior era ligeiramente maior que o istmo posterior, porém sem significância.
No grupo de mulheres com história de cesárea a diferença significativa da espessura entre o istmo anterior e posterior foi observada em todos os casos, o que está de acordo com o relatado na literatura, que observou a redução da espessura da parede anterior nessas mulheres ${ }^{4,6,9,17}$.

Neste estudo, não se encontrou correlação entre o número de cesáreas prévias e o tempo decorrido após a última cirurgia com a redução da espessura do istmo anterior. Esses resultados divergem dos obtidos por Wang et al. ${ }^{15}$, que encontraram que a espessura do istmo anterior era significativamente menor nas mulheres submetidas a múltiplas cesáreas.

De modo geral, os resultados obtidos neste estudo são concordantes com a literatura mais recente sobre o tema no que diz respeito à redução da espessura da parede anterior do segmento inferior uterino nas pacientes submetidas à cesárea.

Este estudo concluiu que a redução da espessura do istmo anterior em relação à parede do istmo posterior uterino é significativa nas pacientes com cesárea prévia e que a ultrassonografia transvaginal constitui um método adequado para a avaliação dessas pacientes.

Este estudo apresenta algumas limitações. A primeira delas é que não há literatura disponível para comparação dos resultados considerando a técnica empregada. Outra limitação é que foram excluídas do estudo as mulheres com útero em retroflexão, que é um importante critério de risco apontado na literatura, juntamente com o número de cesáreas, para cicatrizes com deficiências graves. A inclusão desse grupo de mulheres certamente revelaria um percentual maior de defeitos na cicatriz de histerotomia. Por fim, tratou-se de um estudo com casuística pequena. Futuros estudos com amostras maiores são necessários para avaliar a reprodutibilidade do método proposto neste estudo.

\section{Referências}

1. Mercer BM, Gilbert S, Landon MB, Spong CY, Leveno KJ, Rouse DJ, et al. Labor outcomes with increasing number of prior vaginal births after cesarean delivery. Obstet Gynecol. 2008; 111 (2 Pt 1):285-91.

2. Sufang G, Padmadas SS, Fengmin Z, Brown JJ, Stones RW. Delivery settings and caesarean section rates in China. Bull World Health Organ. 2007;85(10):755-62.

3. Brasil. Ministério da Saúde. Brasil registra aumento no número de cesáreas [Internet]. 2011 [cited 2011 Dec 20]. Available from: http://portal. saude.gov.br/portalsaude/index.cfm?portal=pagina . visualizarNoticia\&codConteudo $=3349 \&$ codModuloArea $=162 \&$ chamada=brasil-registra-aumento-no-numero-de-cesareas

4. Ofili-Yebovi D, Ben-Nagi J, Sawyer E, Yazbek J, Lee C, Gonzalez $J$, et al. Deficient lower-segment cesarean section scars: prevalence and risk factors. Ultrasound Obstet Gynecol. 2008;31(1):72-7.
5. Jauniaux E, Jurkovic D. Placenta accreta: pathogenesis of a 20th century iatrogenic uterine disease. Placenta. 2012;33(4): 244-51.

6. Van der Slikke JW, Bij Vaate M, Brölmann HA, Van der Voet LF, Veersema S, Bartholomew J, et al. Ultrasound evaluation of the caesarean scar: relation between a niche and postmenstrual spotting. Ultrasound Obstet Gynecol. 2010;36(Suppl 1):1 .

7. Santos Filho OO, Nardozza LM, Araújo Junior E, Rolo LC, Camano L, Moron AF. Cesarean uterine scar evaluation by the gray-level histogram. Rev Assoc Med Bras. 2010;56(1):99-102.

8. Surapaneni K, Silberzweig JE. Cesarean section scar diverticulum: appearance on hysterosalpingography. AJR Am J Roentgenol. 2008; 190(4):870-4.

9. Yazicioglu HF. Assessment of cesarean section scars with transvaginal sonography. Ultrasound Obstet Gynecol. 2010;35(2):250-1. 
10. Maymon R, Svirsky R, Smorgick N, Mendlovic S, Halperin R, Gilad K, et al. Fertility performance and obstetric outcomes among women with previous cesarean scar pregnancy. J Ultrasound Med. $2011 ; 30(9): 1179-84$

11. Vilarino FL, Bianco B, Martins ACM, Christofolini DM, Barbosa CP. Endometriose em cicatriz cirúrgica: uma série de 42 pacientes. Rev Bras Ginecol Obstet. 2011 ; 33(3):123-7.

12. Kushtagi P, Garepalli S. Sonographic assessment of lower uterine segment at term in women with previous cesarean delivery. Arch Gynecol Obstet. $2011 ; 283(3): 455-9$.

13. Jastrow N, Chaillet N, Roberge S, Morency AM, Lacasse $Y$, Bujold E. Sonographic lower uterine segment thickness and risk of uterine scar defect: a systematic review. J Obstet Gynaecol Can. 2010;32(4):321-7.

14. Vikhareva Osser O, Valentin L. Clinical importance of appearance of cesarean hysterotomy scar at transvaginal ultrasonography in nonpregnant women. Obstet Gynecol. 2011;117(3):525-32.

15. Wang CB, Chiu WW, Lee CY, Sun YL, Lin YH, Tseng CJ. Cesarean scar defect: correlation between cesarean section number, defect size, clinical symptoms and uterine position. Ultrasound Obstet Gynecol. 2009;34(1):85-9.

16. Martins WP, Barra DA, Gallarreta FM, Nastri CO, Mauad Filho F. Lower uterine segment thickness measurement in pregnant women with previous cesarean section: reliability analysis using two- and three-dimensional transabdominal and transvaginal ultrasound. Ultrasound Obstet Gynecol. 2009;33(3):301-6.

17. Cheung VY. Sonographic measurement of the lower uterine segment thickness: is it truly predictive of uterine rupture? J Obstet Gynaecol Can. 2008;30(2):148-51.

18. Barra DA, Martins WP, Gallarretta FM, Nastri CO, Nicolau LG, Mauad Filho F. Medida da espessura do segmento uterino inferior em gestantes com cesárea prévia: análise da reprodutibilidade intra-e interobservador por ultra-sonografia bi- e tridimensional. Rev Bras Ginecol Obstet. 2008;30(3):142-8.

19. Williams JW, Hirschowitz L. Assessment of uterine wall thickness and position of the vascular plexus in the deep myometrium: implications for the measurement of depth of myometrial invasion of endometrial carcinomas. Int J Gynecol Pathol. 2006;25(1): 59-64.

20. Bérubé L, Arial M, Gagnon G, Brassard N, Boutin A, Bujold E. Factors associated with lower uterine segment thickness near term in women with previous caesarean section. J Obstet Gynaecol Can. $2011 ; 33(6): 581-7$.

21. Uppal T, Lanzarone V, Mongelli M. Sonographically detected caesarean section scar defects and menstrual irregularity. J Obstet Gynaecol. 2011;31(5):413-6. 